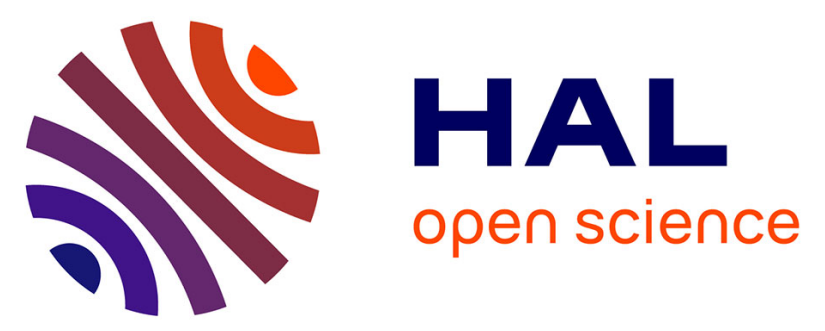

\title{
Quantitative linear and nonlinear Resonance Inspection Techniques and Analysis for material characterization: Application to concrete thermal damage.
}

Cedric Payan, T.J. Ulrich, P.Y. Le Bas, T.A. Saleh, Maria Guimaraes

\section{To cite this version:}

Cedric Payan, T.J. Ulrich, P.Y. Le Bas, T.A. Saleh, Maria Guimaraes. Quantitative linear and nonlinear Resonance Inspection Techniques and Analysis for material characterization: Application to concrete thermal damage.. Journal of the Acoustical Society of America, 2014, 136 (2), pp.537-546. 10.1121/1.4887451 . hal-01044669

\author{
HAL Id: hal-01044669 \\ https://hal.science/hal-01044669
}

Submitted on 24 Jul 2014

HAL is a multi-disciplinary open access archive for the deposit and dissemination of scientific research documents, whether they are published or not. The documents may come from teaching and research institutions in France or abroad, or from public or private research centers.
L'archive ouverte pluridisciplinaire HAL, est destinée au dépôt et à la diffusion de documents scientifiques de niveau recherche, publiés ou non, émanant des établissements d'enseignement et de recherche français ou étrangers, des laboratoires publics ou privés. 
Quantitative linear and nonlinear Resonance Inspection Techniques and Analysis for material characterization: Application to concrete thermal damage.

\author{
C. Payan ${ }^{1}$, T.J. Ulrich ${ }^{2}$, P.Y. Le Bas ${ }^{2}$, T. Saleh ${ }^{3}$, M. Guimaraes ${ }^{4}$ \\ ${ }^{1}$ Aix Marseille Univ, LMA UPR CNRS 7051, Marseille France \\ ${ }^{2}$ Los Alamos National Laboratory, EES-17, Los Alamos NM USA \\ ${ }^{3}$ Los Alamos National Laboratory, MST-16, Los Alamos NM USA \\ ${ }^{4}$ Electrical Power Research Institute, Charlotte NC US
}

\begin{abstract}
:
Developed in the late 80's, Nonlinear Resonant Ultrasound Spectroscopy (NRUS) has been widely employed in the field of material characterization. Most of the studies assume the measured amplitude to be proportional to the strain amplitude which drives nonlinear phenomena. In 1D resonant bar experiments, the configuration for which NRUS was initially developed, this assumption holds. However, it is not true for samples of general shape which exhibit several resonance mode shapes. This paper proposes a methodology based on linear resonant ultrasound spectroscopy, numerical simulations and nonlinear resonant ultrasound spectroscopy to provide quantitative values of nonlinear elastic moduli taking into account the 3D nature of the samples. In the context of license renewal in the field of nuclear energy, this study aims at providing some quantitative information related to the degree of micro-cracking of concrete and cement based materials in the presence of thermal damage. The resonance based method is validated as regard with concrete microstructure evolution during thermal exposure.
\end{abstract}

(C) 2013 Acoustical Society of America.

PACS numbers: 43.25.Ba, 43.25.Gf 


\section{Introduction}

\section{A. Context}

Many Non-Destructive Evaluation (NDE) methods such as thermography, radiography, electrical resistivity, radar, etc. provide information about the state of the concrete but the only one which is directly related with concrete mechanical characteristics is acoustics. Standard methods such as ISO 1920-7 and ASTM C597-09, founded on low frequency $(<100 \mathrm{kHz}=$ wavelength $>4 \mathrm{~cm})$ pulse wave velocity, cannot provide information about the presence of a localized defect at the centimeter scale. Developments in the field of concrete NDE show that the information obtained from the nonlinear elastic wave propagation can increase the sensitivity by a factor 10 or more in homogeneous materials ${ }^{1}$ and in concrete and cement based materials ${ }^{2}$.

In the context of license renewal in the field of nuclear energy, maintaining in service concrete structures for the period of long-term operations is challenging. For ecologic, economic and societal reasons, replacing a structure is often complicated. Subjected to radiation and medium temperature $\left(<500^{\circ} \mathrm{C}\right)$ for a long period of time, the integrity of concrete in the pedestal and biological shield wall in nuclear plants and the overpack of storage casks remains unknown. Thus, increasing the safety and anticipating concrete degradation through the use of a powerful tool, with the capability to characterize concrete mechanical quality as its main function, should be welcome. The purpose of this study is to provide quantitative information about the amount of damage in concrete subjected to thermal damage.

\section{B. Thermal damage of concrete}

Chemical reaction occurring with thermal damage process of concrete is known. Above $105^{\circ} \mathrm{C}$, all the free and a part of adsorbed water are released. From 180 to $300^{\circ} \mathrm{C}$, hydrated silicates decompose by tearing water molecules, which evaporate. Above $450^{\circ} \mathrm{C}$, the portlandite breaks down and releases water: $\mathrm{Ca}(\mathrm{OH})_{2} \rightarrow \mathrm{CaO}+\mathrm{H}_{2} \mathrm{O}$. The first aggregate transformation ( $\alpha$ to $\beta$ quartz transition) appears at $600^{\circ} \mathrm{C}$. Other transformations occur up to the aggregates/cement paste fusion at about $1300^{\circ} \mathrm{C}$. This process results in an increase of porosity and micro-cracking.

This study focuses on temperatures encountered in nuclear facilities so up to $400^{\circ} \mathrm{C}$. In this range, three sets of four samples (about $6 \times 10 \times 10 \mathrm{~cm}^{3}$ ) were manufactured by $\mathrm{CSTB}^{3}$ (Centre Scientifique et Technique du Bâtiment, France). Their compositions are given in Table 1. For each set, one sample is kept as a reference, the second one is damaged at $120^{\circ} \mathrm{C}$, the third one at $250^{\circ} \mathrm{C}$, the last at $400^{\circ} \mathrm{C}$ (refer to the codification in Table 1). Thermal damage is achieved by heating the sample at the desired temperature with an increase of $0.5^{\circ} \mathrm{C} / \mathrm{min}$, holding a constant temperature for 3 hours, then decreasing by $0.5^{\circ} \mathrm{C} / \mathrm{min}$. The thermal damage protocol was designed to avoid any undesired mechanical damage induced by thermal gradients, thus internal stresses, during heating. In concrete the most brittle zone is the interface between aggregates and cement paste. This zone, namely Interfacial Transition Zone (ITZ), is the most porous and crystallized region.

The average Coefficient of Thermal Expansion (CTE) of aggregates (mainly limestone in this study) ranges between $5.5 \times 10^{-6}<\mathrm{CTE}_{\mathrm{A}}<11.8 \times 10^{-6}{ }^{\circ} \mathrm{C}^{-1} 3$. The average hardened cement paste CTE ranges between $11 \times 10^{-6}<\mathrm{CTE}_{\mathrm{P}}<20 \times 10^{-6}{ }^{\circ} \mathrm{C}^{-1}{ }^{4}$. Considering a single inclusion in a matrix, numerical simulation ${ }^{5}$ shows that in such a case $\left(\mathrm{CTE}_{\mathrm{A}}<\mathrm{CTE}\right)$, most of the damage will occur at the ITZ. Considering the spatial distribution of aggregates, numerical simulation ${ }^{6}$ shows that depending on the spatial arrangement, on the shape and on the size distribution of 
aggregates, a complex thermo mechanical interplay between them occurs. As $\mathrm{CTE}_{\mathrm{A}}<\mathrm{CTE}_{\mathrm{P}}$, the most affected region will also be the ITZ.

In concrete, both $\mathrm{CTE}_{\mathrm{A}}$ and $\mathrm{CTE}_{\mathrm{P}}$ are temperature dependent. $\mathrm{CTE}_{\mathrm{A}}$ increases with temperature up to $17 \times 10^{-6}$. When increasing temperature ${ }^{3}$ above $150^{\circ} \mathrm{C}$, the cement paste starts shrinking while the aggregates are still expanding. In such a case radial cracks (cracks connection between two neighbor aggregates) should also appear in the cement paste. Taking into account the fact that the ITZ is the most brittle zone, it is reasonable to assume that most of the damage will occur in this region with some additional cracks inside the matrix. The nature of thermal damage also allows considering a homogeneous and isotropic damage.

\section{Nonlinear mesoscopic behavior of concrete}

The nonlinear elastic behavior of homogeneous material is described by the Landau and Lifshitz ${ }^{7}$ elasticity theory. In the 1990's, authors ${ }^{8}$ report the so called "non-classical" nonlinear behavior of complex materials such as rocks and concrete which do not follow the former theory. Phenomena, such as large and unexpected nonlinearity, hysteresis and endpoint-memory effects are reported whether under quasi-static or dynamic loading ${ }^{8}$. To describe this complex behavior, authors introduce a $1 \mathrm{D}$ phenomenological nonlinear and hysteretic modulus ${ }^{9}$ :

$K=K_{0}\left[1-\beta \varepsilon-\delta \varepsilon^{2}-\cdots-\alpha(\Delta \varepsilon, \dot{\varepsilon})\right]$,

where $K_{0}$ is the elastic modulus, $\beta$ and $\delta$ are the Landau type nonlinear elastic terms, $\alpha$ is the phenomenological nonlinear hysteretic parameter, the dot indicates the time derivative, $\Delta \varepsilon$ is the strain amplitude. Physically, the first nonlinear terms relate the nonlinearity of the force/displacement relationship at the atomic scale. The physical origin of the last one arises at the mesoscopic scale. Phenomena such as opening/closing of microcracks, break/recovery of cohesive grain bonds properties, friction, contacts, capillary effects in porosities, etc., are expected to be responsible of the complex behavior of this nonlinear mesoscopic class of materials into which concrete falls. Even if there is still not any universal theory allowing to physically describing this class of materials, the nonlinear $\alpha$ parameter can nevertheless be employed to quantify the nonlinearity and thus used for nondestructive evaluation. Several methods were used to evaluate the nonlinearity. In concrete, among others, one can cite Nonlinear Resonant Ultrasound Spectroscopy (NRUS) which is employed by Abeele et al. ${ }^{10}$ and Bentahar et al. ${ }^{11}$ to study the effect of mechanical damage on the measured nonlinearity. Bouchaala et al. ${ }^{12}$ reports the sensitivity of the nonlinear parameter to carbonation. Lesnicki et al. ${ }^{13}$ studies the influence of Alkali Silicate Reaction (ASR). Payan et al. ${ }^{2}$ qualitatively shows the effect of thermal damage on the nonlinearity using pressure and shear transducers. These studies were conducted with various resonance modes but all of them only provide qualitative variation of the nonlinearity with increasing the amount of damage. These qualitative data do not allow comparisons to be done among a large variety of material and applications.

While the physical mechanisms responsible of the nonlinear behavior are still not understood, the aim of this paper is to propose a nonlinear resonance based method able to provide quantitative information, and thus, to allow proper comparisons and expectation of possible mechanisms to be done.

\section{Resonance Inspection Techniques and Analysis \\ A. Introduction to the methodology}


NRUS consists of conducting resonance frequency measurements at various driving amplitudes (Fig.2). For a given resonance mode, a linear material maintains a constant resonance frequency while a nonlinear mesoscopic material softens with increasing drive amplitudes (i.e., dynamic strain). Following Eq.1, this softening results in a decrease of the resonant frequency as a function of the strain amplitude $(\Delta \varepsilon)$. The nonlinear $\alpha$ parameter is obtained by the linear decrease of the resonance frequency indicating that the non-classical phenomenon dominates the global nonlinear behavior ${ }^{14}$ :

$\Delta f / f_{0}=\alpha \Delta \varepsilon$,

where $f_{0}$ is the low amplitude linear resonance frequency and $\Delta f=f-f_{0}$ ( $\mathrm{f}$ is the resonance frequency for increasing drive amplitudes).

This method quantitatively applies to samples with appropriate 1D geometry (cylinder with large aspect ratio), as the longitudinal vibration mode is very easy to detect, the measured vibration amplitude of the sample is proportional to the strain amplitude [Fig.1(a)]. However, for more complex geometry [Fig.1(b)], this assumption is no longer valid because of the complexity of mode shapes. That point is underlined by Johnson and Sutin ${ }^{15}$, who were unable to evaluate the nonlinear parameter for a parallelepiped type sample using NRUS.

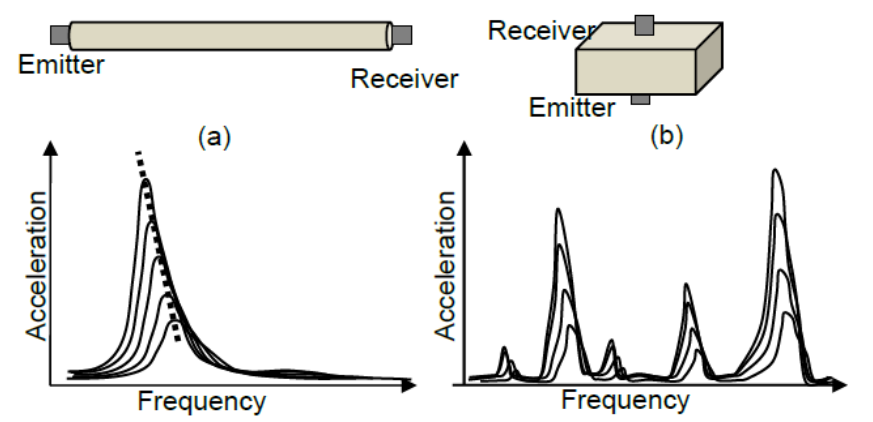

FIG. 1. NRUS curves for (a) optimal and (b) more complex geometry.

To overcome this difficulty, in order to evaluate the strain amplitude for a given mode shape, a methodology based on Resonant Ultrasound Spectroscopy (RUS) and numerical simulation is developed. The steps of the method consist on:

- The geometry and the density of the sample serve as input for a linear resonance method which provides the linear elastic tensor.

- The elastic tensor serves as an input for a numerical simulation which allows the experiment, including the effect of the emitters and receivers mass, to be modeled. This step allows linking the quantity measured by the receiver to the strain amplitude inside the sample, as well as identifying the mode shape under study.

- The mode shape selected and the strain determined, the NRUS experiment provides an absolute value of the nonlinearity.

This method is detailed in the following.

\section{B. Resonant Ultrasound Spectroscopy}

Adapted to complex 3D geometries, resonance inspection techniques are known and have been employed for years ${ }^{16}$. Resonant Ultrasound Spectroscopy (RUS) allows the material elastic properties to be determined accurately by non-destructive means. The input values are the sample geometry and the density. By exciting the sample over a large frequency range, one can extract the resonance peaks (experimentally measured values) corresponding to 
various eigenmodes. Then, by combining experimental and input values, an inversion algorithm provides the full elastic tensor of the sample. This can apply to any elastic material type (isotropic or anisotropic). RUS has been employed in various homogeneous materials and more recently in inhomogeneous ones such as rocks ${ }^{17}$ and cement ${ }^{18}$. Here the application of RUS is extended to various forms of concrete materials.

The procedure for performing a RUS measurement and obtaining the full elastic tensor from such a measurement is well defined in general ${ }^{16}$, with specifics for dealing with inhomogeneous earth materials having been specified by Ulrich et al. ${ }^{17}$ and recently for anisotropic and highly damped material such as bone ${ }^{19}$. As such, the reader is refereed to these publications for details of the method. Here the experimental system and an example spectrum are briefly presented before focusing on the results as a function of thermal damage.

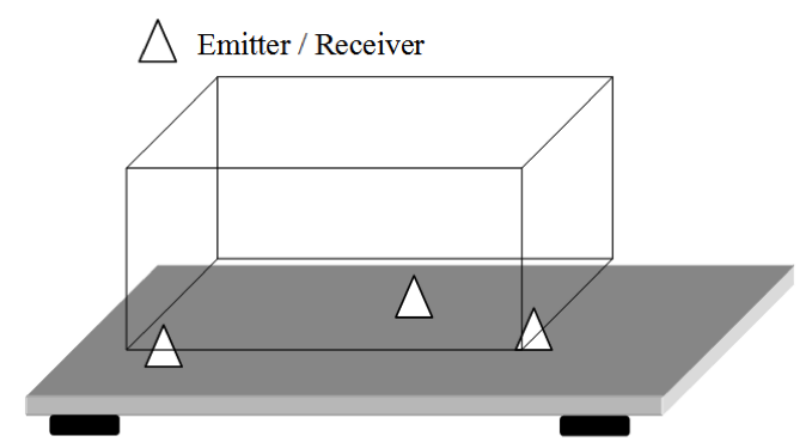

FIG. 2. Schematic of rectangular concrete block sample sitting on the conical transducer stand.

Traditional RUS requires free boundary conditions in order to invert the measured frequencies to find the elastic moduli using the Visscher RUS algorithm ${ }^{16}$. To approximate this, the sample is placed upon a stand containing 3 conically shaped transducers (FIG. 2). Dry contact is used, however, use of an ultrasonic coupling gel may also be applied to the transducer/sample contact area without adverse effects. Two of the available transducers are used during the measurement: one as an emitter and one as a receiver. The order/placement of these transducers has minimal importance as resonance frequencies will not change with the location of the source/receiver, however, the amplitude of the resonance frequencies will change. As the amplitude is not used in the inversion for elastic moduli, this is unimportant as long as each resonance frequency is large enough to be measured. Positioning transducers near corners of the sample and/or avoiding points of symmetry is sufficient to avoid nodal locations for the low lying modes.

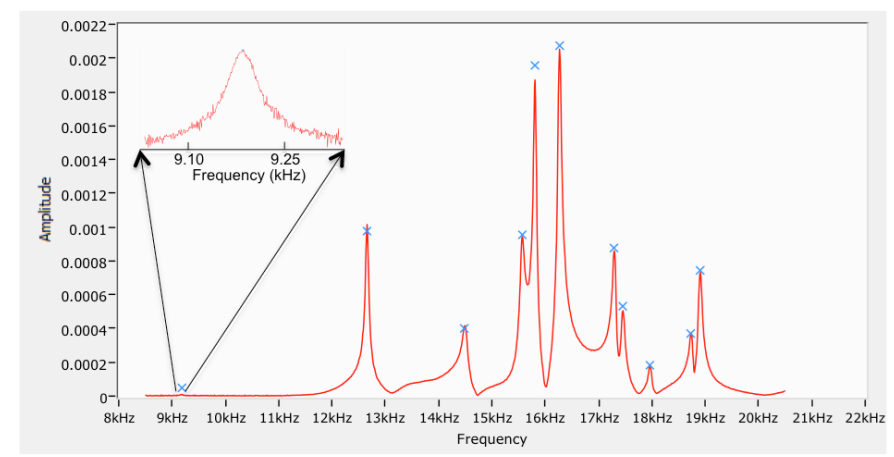

FIG. 3. (Color Online) Resonance spectrum taken from sample OC-120. 'x' indicates peak values used in RUS inversion. 
After placement onto the stand, the sample is excited (here using a National Instrument PXI5406 function generator) in a constant amplitude stepped sine fashion and the resulting amplitude recorded at each frequency is recorded by the receiver and digitized (here using a National Instrument PXI-5122 A/D high speed digitizer). Both generation and acquisition lines are managed by the RITA@ (Resonance Inspection Techniques and Analysis) software, designed and implemented by the LANL Geophysics group's Wave Physics team (Ulrich and Le Bas). An example spectrum is shown in FIG. 3 as measured from the OC-120 sample. Note the x's indicating the frequency values used in the RUS inversion for this sample. Once a sufficient number of resonance peaks have been identified and measured, the inversion can be performed to extract the elastic moduli. For all samples herein 11 resonance peaks were measured and an inversion was performed using an isotropic assumption. This resulted in the inversion being performed to better than 1\% RMS error (deviation between experimental and computed resonance frequencies) for all samples with the exception of HPC 250 whose inversion was obtained to only 3\% RMS error. This increased RMS error may be due to either overwhelming inhomogeneity over this scale or an incorrect assumption in the elastic tensor symmetry (i.e., sample may be anisotropic).

\section{Nonlinear Resonant Ultrasound Spectroscopy and numerical simulation}

The goal of the proposed method is to identify a mode similar to the Young's mode for a cylinder and so for which Eq. 2 applies. Here we have chosen a bulk/breathing mode. The experimental scheme is presented in FIG. 4. Generation and acquisition are ensured by the same equipment than for RUS section. The generation is coupled to a voltage amplifier TEGAM 2350. Both generation and acquisition lines are also managed by the RITA@ software, NRUS module. For NRUS studies, a transducer able to drive the sample at high amplitude is needed. An ultrasonic cleaning transducer (Ultrasonics World, Delaware, USA) is driven by the amplifier up to a peak to peak voltage of $400 \mathrm{~V}$. To minimize the influence of this big transducer on the bulk resonance mode, it is glued at the sample center. A Polytec laser vibrometer (OFV 5000, $1.5 \mathrm{MHz}$ bandwidth) records the out of plane particle velocity at the same location on the side opposite. This arrangement is chosen in order to favor the measurement of the bulk mode and limit the sensitivity to flexural/shear modes.

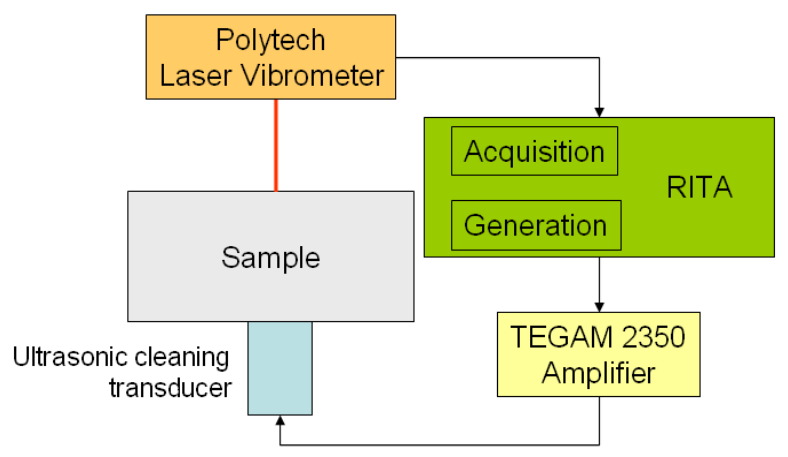

FIG. 4. (Color Online) NRUS experimental scheme.

To perform quantitative NRUS, the protocol is as follows:

Step 1. Experimental resonance spectrum: the sample is excited at low amplitude over a large frequency band in order to identify the resonance modes of the sample. An example of resonance curves is given in FIG. 5 for the M samples. 


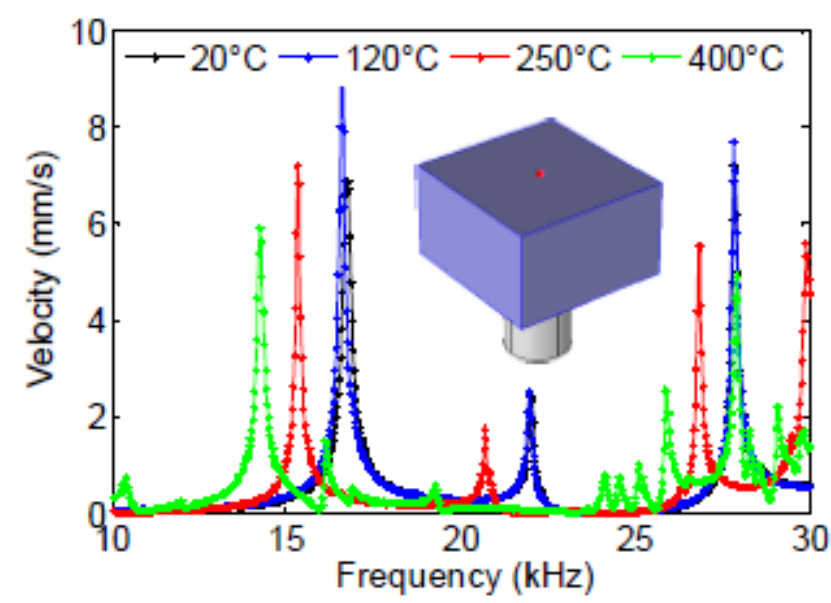

FIG. 5. (Color Online) Resonance modes of the Mortar samples set. The sample is also shown, including the transducer and the laser spot location (spot at the sample center).

The peaks around $15 \mathrm{kHz}$ present the most energetic modes in this configuration, i.e., generation with the transducer at the sample center, acquisition at the side opposite center (FIG. 5). To link the measured particle velocity to the strain inside the sample, the experiment is modeled by finite element numerical simulation.

Step 2. Identification of resonance modes: The linear elastic characteristics of the samples are used as input in the numerical simulation using Comsol ${ }^{\circledR}$, Solid Mechanics module assuming homogeneous and isotropic material. An example for the M20 sample is given in Fig. 6 in the $10-30 \mathrm{kHz}$ frequency range. In this range, numerical simulation provides the resonance modes corresponding to the experiments, i.e., including the ultrasonic cleaning transducer attached to the sample. The bulk mode is identified around $22 \mathrm{kHz}$ (FIG. 6). For the latter, one can link the measured velocity amplitude $\Delta v_{\text {laser }}$ to the volumetric strain amplitude $\Delta \varepsilon$ by:

$$
\Delta \varepsilon=C \Delta v_{\text {laser }}
$$

where $C$ is a constant depending on the linear elastic properties of the sample. This constant is evaluated at the resonance frequency by the average volumetric strain amplitude in the sample divided by the out of plane velocity amplitude at the laser spot. This quantity has been checked to be amplitude independent in simulations; meaning that in experiments, multiplying the velocity amplitude (laser measurement) by this constant provides the volumetric strain amplitude. For each sample, the bulk mode is identified and the $C$ constant is determined. The data summary is presented in Table 2. 


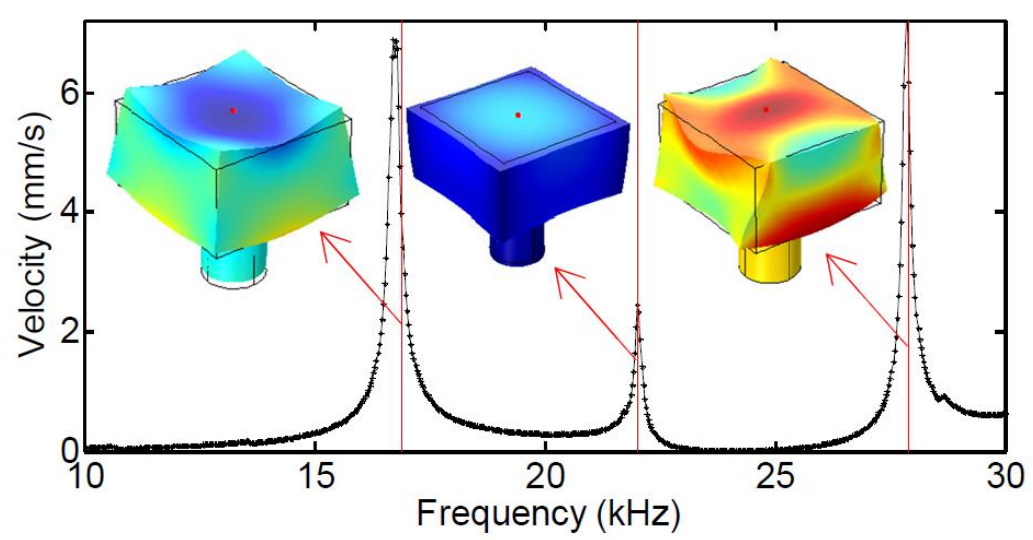

FIG. 6. (Color Online) Experimental and simulated resonance modes for M20 sample. The curve is the experimental spectrum, the vertical lines are the simulated resonant frequencies (limited to the most three energetic ones). The corresponding mode shapes are also shown.

Table 2 shows that the accuracy of the method decreases with increasing damage. This can be explained by the decrease of linear elastic properties which effects the position of the resonance peaks whereby the bulk mode is influenced more by nearby resonances.

Step 3. Performing NRUS: With the bulk mode determined, the sample is driven around the corresponding frequency. The NRUS resonance curves are obtained by sending tone-burst series to the transducer starting from $f_{l}$ to $f_{n}$ (FIG. 7), with a constant $\Delta f$ step, at a constant amplitude $A_{l}$. For each tone-burst, the received amplitude is determined from a heterodyne Fourier analysis at the driving frequency of the received signal. The driving amplitude is increased by a constant step $\Delta A$ from $A_{1}$ to $A_{n}$ then the tone burst series starts again. The number of driving periods of the tone-burst is determined so as to exceed the quality factor $Q$ of the sample in order to ensure steady state conditions. The highest quality factor over the full set of sample was set to ensure steady state conditions for every sample (about 200 for present samples). The quality factor is defined as the resonance frequency divided by the resonance peak width evaluated at the half maximum amplitude for a given mode.

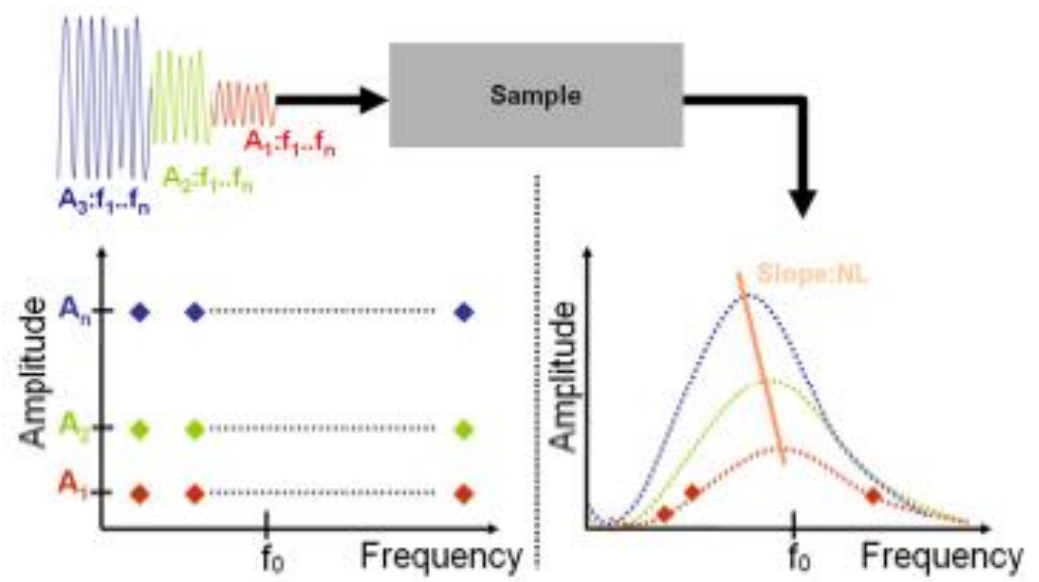

FIG. 7. (Color Online) Data acquisition procedure. Left panel: generation. Right panel: reception

The slope of the line FIG. 7 is proportional to the nonlinearity. The absolute nonlinear $\alpha$ parameter is evaluated by the combination of Eq. 2 and Eq.3. The linearity of the experimental 
system is checked by applying NRUS to a known linear material (Plexiglas) (FIG. 8) with the same geometry as the concrete samples and thus exhibiting the same resonance mode shapes.

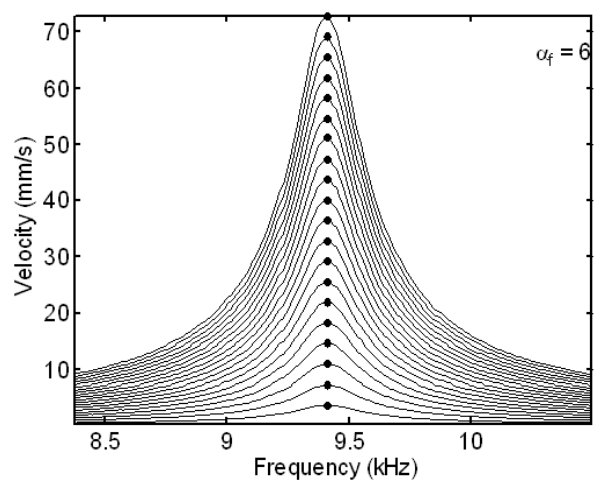

FIG. 8. Plexiglas sample NRUS results

\section{Results and Discussion}

\section{A. RUS}

The results for all samples are shown in Fig. 9. These results highlight the global decrease of the Young modulus and Poisson ratio with increasing thermal damage. Due to the presence of adjuvant in HPC, The Young's modulus of HPC is greater than OC. The residual Young's modulus is about $50 \%$ at $400^{\circ} \mathrm{C}$ for both $\mathrm{HPC}$ and OC. These values are in agreement with the literature $^{20,21}$ with the same kind of damage protocol (slow increase and decrease of temperature) and limestone type aggregates. The $\mathrm{M}$ samples are less affected than HPC and OC ones with a $71 \%$ residual Young's modulus at $400^{\circ} \mathrm{C}$. The Poisson ratio decreases with temperature by an amount in agreement with literature ${ }^{22}$.

\begin{tabular}{|c|c|c|}
\hline Sample & $E$ (Gpa) & $v$ \\
\hline \multicolumn{2}{|c|}{ High Performance Concrete } \\
\hline HPC 20 & 48.9 & 0.21 \\
\hline HPC 120 & 45.3 & 0.19 \\
\hline HPC 250 & 39.4 & 0.2 \\
\hline HPC 400 & 24.8 & 0.1 \\
\hline \multicolumn{2}{|c|}{ Ordinary Concrete } \\
\hline OC 20 & 36.9 & 0.2 \\
\hline OC 120 & 35.5 & 0.18 \\
\hline OC 250 & 28.6 & 0.13 \\
\hline OC 400 & 16.4 & 0.08 \\
\hline \multicolumn{3}{|c|}{ Mortar } \\
\hline M 20 & 37.1 & 0.17 \\
\hline M 120 & 38.4 & 0.17 \\
\hline M 250 & 33.6 & 0.14 \\
\hline M 400 & 26.5 & 0.09 \\
\hline
\end{tabular}
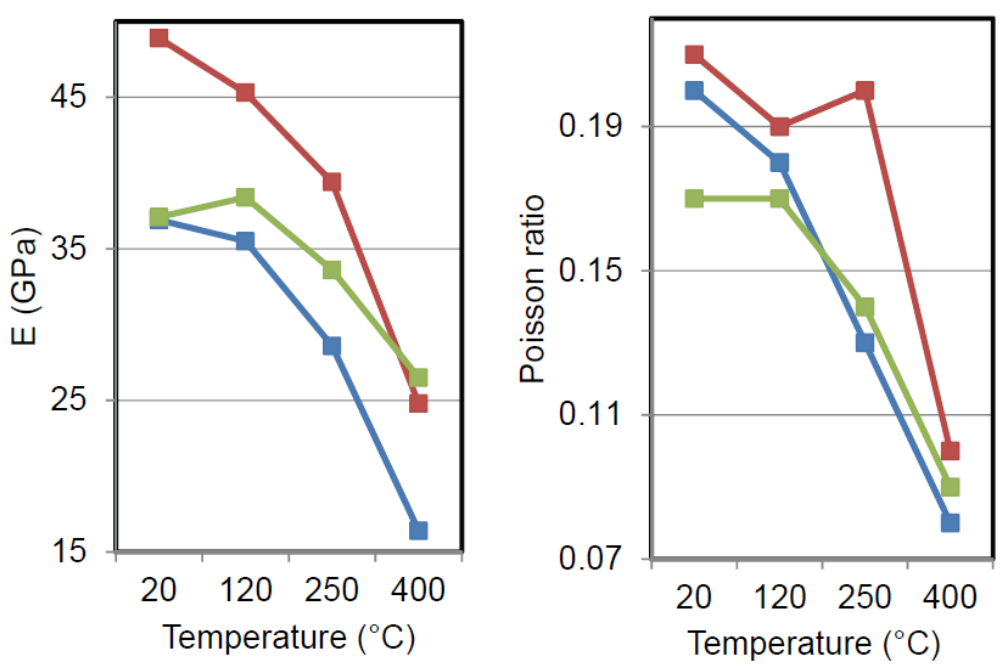

FIG. 9. (Color Online) Evolution of elastic properties for the full set of sample as a function of thermal damage.

\section{B. Quantitative NRUS}


The methodology is applied to each sample. FIG. 10-12 present the full set of results for HPC, OC and M samples, respectively.
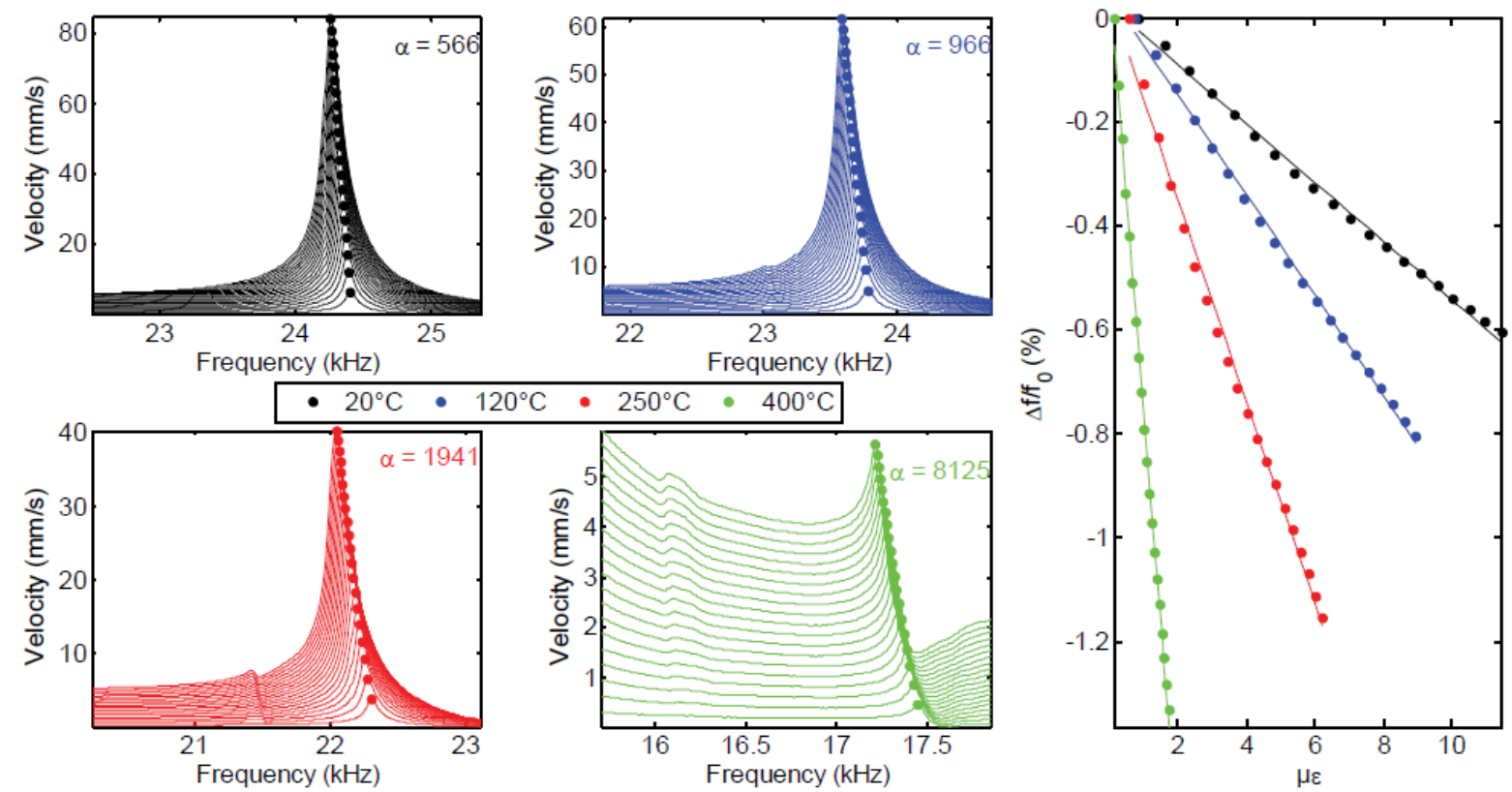

FIG. 10. (Color Online) High Performance Concrete NRUS curves
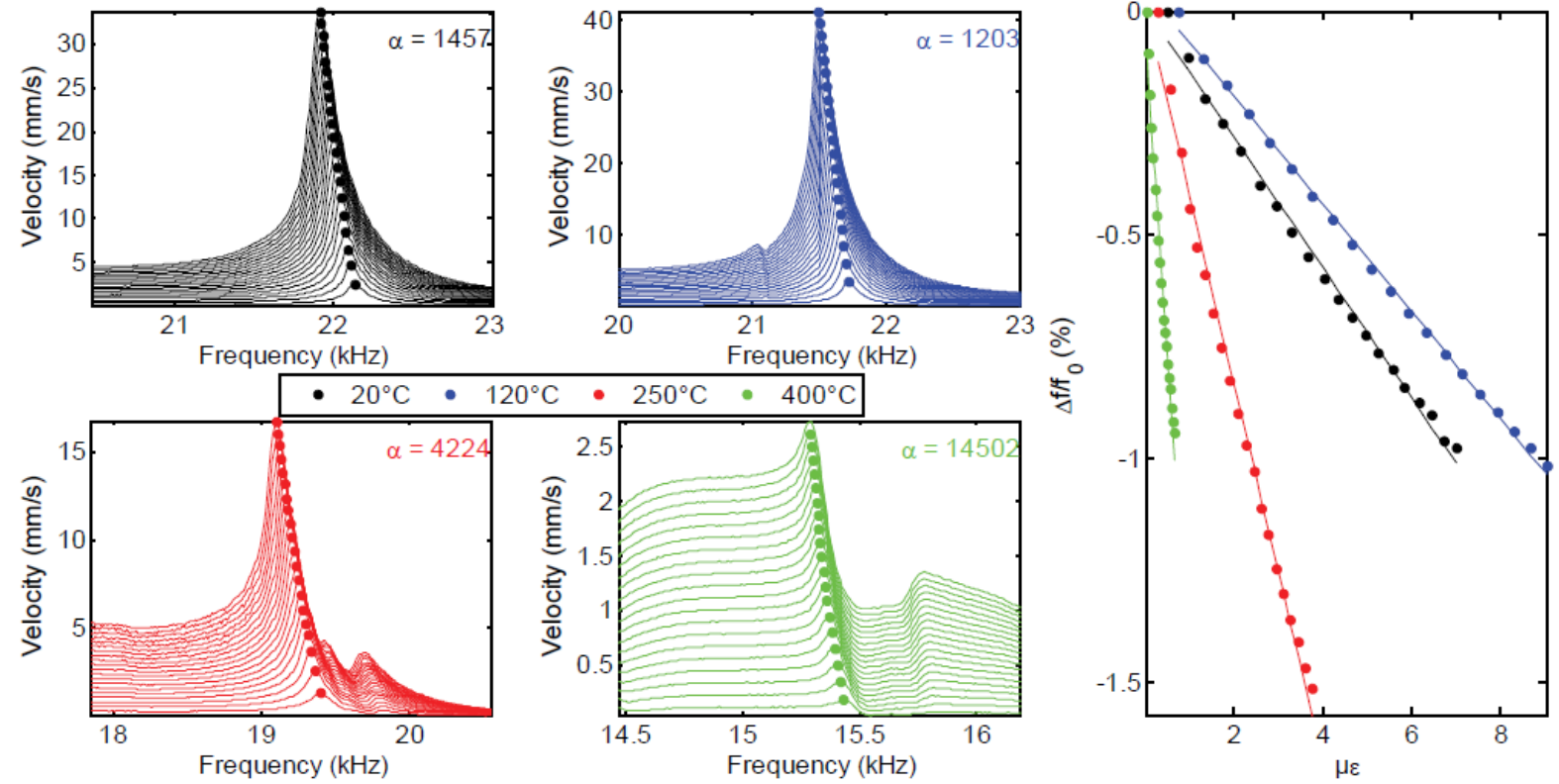

FIG. 11. (Color Online) Ordinary Concrete NRUS curves 

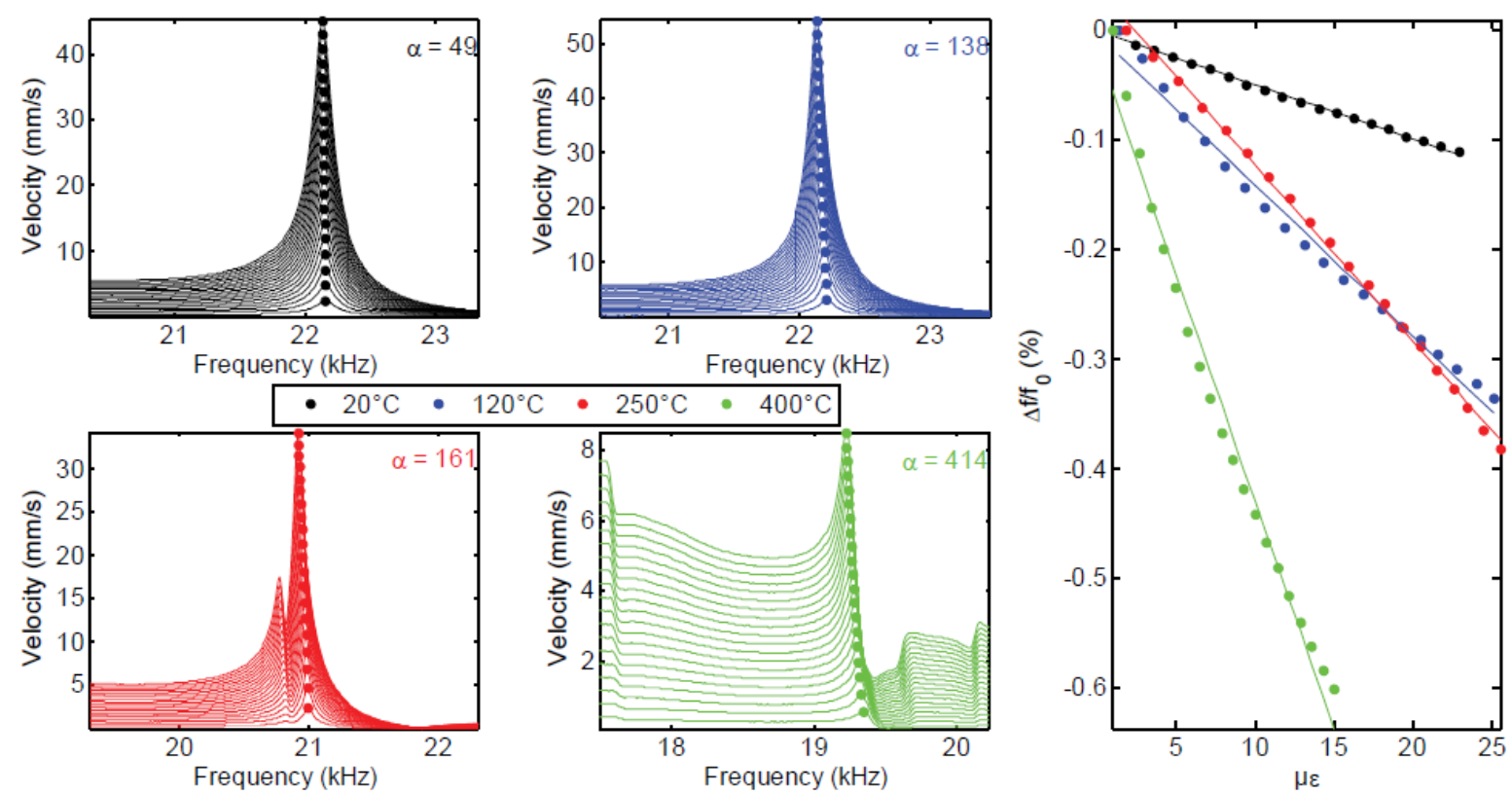

FIG. 12. (Color Online) Mortar NRUS curves

The compilation is provided in FIG. 13.

\begin{tabular}{|c|c|}
\hline Sample & $\alpha$ \\
\hline $\begin{array}{c}\text { High Performance } \\
\text { Concrete }\end{array}$ \\
\hline HPC 20 & 566 \\
\hline HPC 120 & 966 \\
\hline HPC 250 & 1941 \\
\hline HPC 400 & 8125 \\
\hline Ordinary Concrete \\
\hline OC 20 & 1457 \\
\hline OC 120 & 1204 \\
\hline OC 250 & 4224 \\
\hline OC 400 & 14502 \\
\hline \multicolumn{2}{|c|}{ Mortar } \\
\hline M 20 & 49 \\
\hline M 120 & 138 \\
\hline M 250 & 161 \\
\hline M 400 & 414 \\
\hline
\end{tabular}

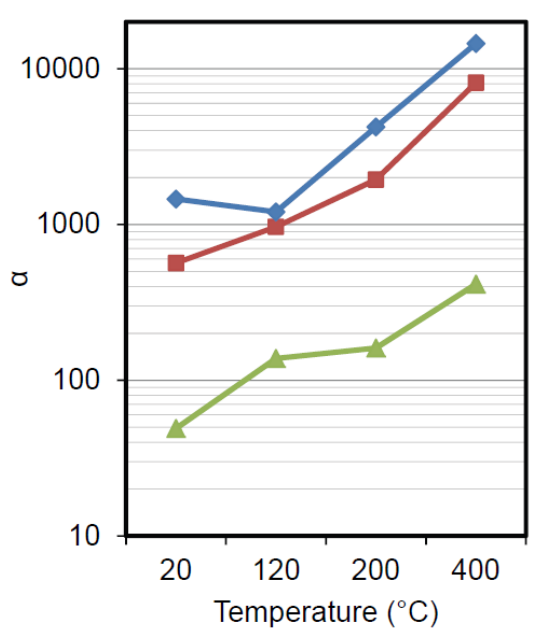

FIG. 13. (Color Online) Result's compilation of nonlinear parameters.

Several interpretations and comments arise from these data.

(i) Comparison with other materials

This quantitative measure allows to compare the actual absolute nonlinear parameter to the literature. These results range in the absolute values reported in rocks ${ }^{23,24}$ under various conditions. In undamaged state, the $\mathrm{M}$ nonlinearity is comparable to alumina, quartzite and cracked pirex ${ }^{15}$. It is worth noticing that except for undamaged mortar, present values are an order magnitude higher than materials such as aluminum or bone $\mathrm{e}^{25}$. These four references are the only ones providing absolute values of the nonlinear parameter. It is thus anticipated to draw general conclusions about possible mechanisms responsible of the nonlinear hysteretic behavior.

(ii) Correlation with the microstructure 
From the thermo-mechanical properties of concrete presented section I.B, the ITZ is identified as an important contributor to the thermal damage process. From the composition of present samples (Tab.1), a rough estimate of the ITZ area can be achieved assuming aggregates as spheres with diameters corresponding to the average aggregate size weighted by the quantity of each class. For M samples, the average ITZ area is $A_{M}=16 \mathrm{~mm}^{2}$. For HPC, which contains also plasticizer and presents the same w/c ratio than $M$ samples, the ITZ area is $A_{H P C}=203 \mathrm{~mm}^{2}$. The ratio of both quantities provides $A_{H P C} / A_{M}=12.7$. On the other hand, the ratio of the nonlinearity for these two samples is $\alpha_{\mathrm{HPC}} / \alpha_{M}=11.5$. This ratio is more or less constant over full the damage process (Fig.13). This rough estimate underlines that the ITZ can be one of the main nonlinearity source in concrete subjected to thermal damage. Due to different composition, the same assumption qualitatively holds for OC samples compared to M ones.

It is important to notice that taking into account the volumetric density of ITZ, the results are opposite to what is expected. Due to the small diameters of inclusions, $M$ samples exhibit a density of ITZ considerably higher than HPC. Recent results in plaster ${ }^{26}$ and in concrete ${ }^{27}$ show that the sizes of inclusions (respectively gypsum crystals and aggregates) play a key role on the nonlinear behavior.

The presence of plasticizer in HPC makes the spatial arrangement of aggregates homogeneously distributed. Silica fume allows a better adherence between aggregates and cement paste. Regarding the thermal damage process of concrete described Section I.B, these two observations may explain their lower nonlinearity compared to OC ones.

(iii) Correlation with linear elastic properties

The nonlinear parameter is not correlated with the Young's modulus. Over the full damage process, M exhibits a low nonlinearity compared with ordinary and HPC while its linear elastic properties range between the latters (Fig. 9). The nonlinear parameter is supposed to be physically driven by nonlinear and hysteretic phenomena such as contact, friction, opening/closing of micro cracks at mesoscopic scale. The linear elastic properties are affected by the composition as well as any kind of porosities (crack type or air voids).

(iv) Other physical mechanisms in play

It is worth noticing that actual experiments were performed under ambient laboratory conditions (air conditioning at $20^{\circ} \mathrm{C}$ ), meaning that the influence of water saturation and temperature during experiments is not taken into account here. Water saturation is known to have an influence on the nonlinear response of mesoscopic materials ${ }^{24}$. Temperature changes during experiments are known to be a source of uncertainties for weekly nonlinear materials ${ }^{25}$ (which is not the case here except for M20 sample). This effect can be reduced using an experimental protocol developed by Haupert et al. ${ }^{25}$.

A sensitivity study is provided FIG. 14. For a comparison purpose, the sensitivity of the compressional wave velocity estimated from time of flight measurement is also shown. The nonlinear parameter globally increases by $1000 \%$ while the speed of sound reaches only $25 \%$. The equivalent nonlinearity values found for intact and $120^{\circ} \mathrm{C}$ damaged $\mathrm{OC}$ samples are not explainable except by the sample variability at manufacturing or unexpected change in temperature or humidity during the OC20 experiment. Here, the supposed intact sample is suspected to be too nonlinear making the relative sensitivity of OC samples a little bit lower than the HPC samples. Physically, due to the ITZ effect comparable for both OC and HPC, this sensitivity should be comparable (about 1300\%). 

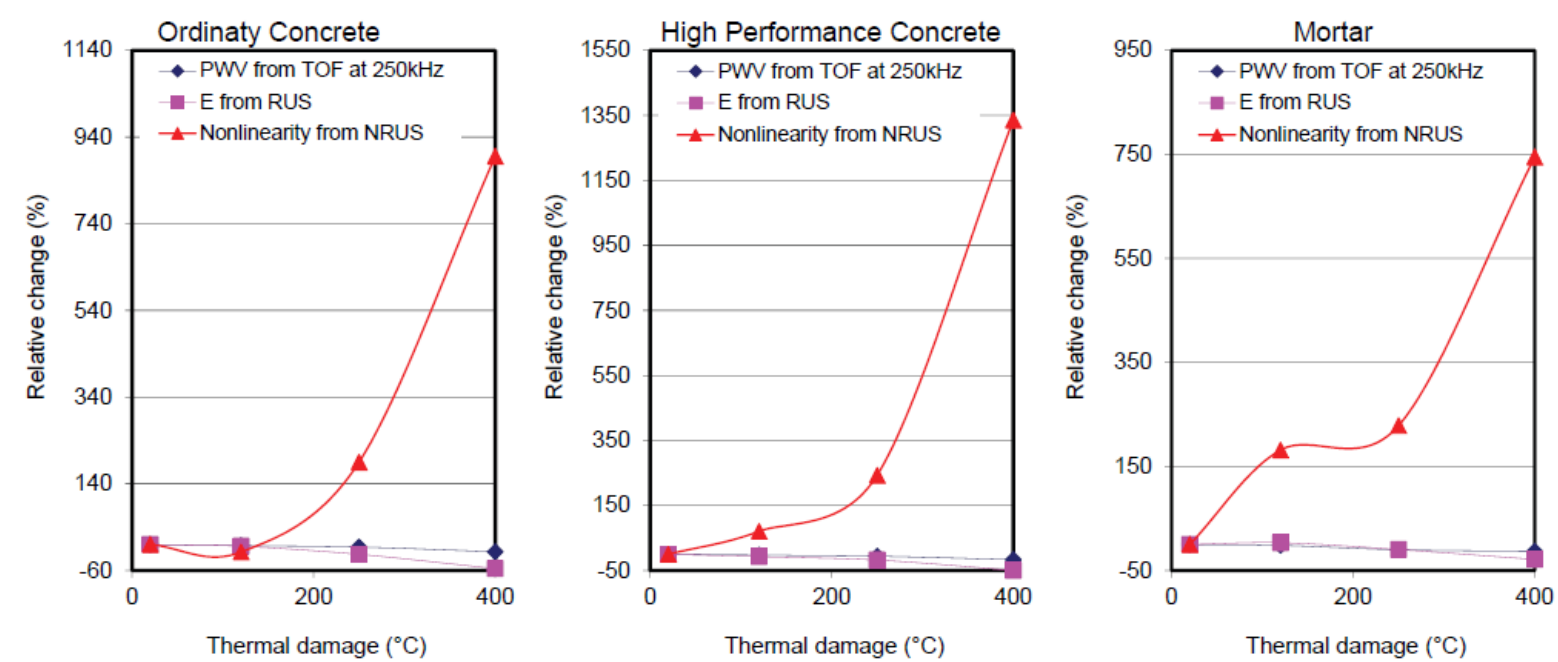

FIG. 14. (Color Online) Sensitivity study. PWV is the Pulse Wave Velocity evaluated from Time Of Flight measurements.

Due to the global fall of elastic properties, one can observe from FIG. 10 to FIG. 12 that the mode density increases around the selected mode for most damaged samples (250 and 400 $\left.{ }^{\circ} \mathrm{C}\right)$. It may become problematic with highly damaged samples for which modes could overlap making impossible the evaluation of the strain amplitude, however, with a different choice of the sample geometry (if possible) one can alleviate this problem. It is observed that the intact state deviation between M, HPC and OC samples remains more or less constant over the full damage process. For lower frequencies, the accuracy would probably have been better but the aim of this paper is to study the bulk mode nonlinearity and highlight the importance of the knowledge of mode shape to properly evaluate the strain amplitude, thus the nonlinearity.

With increasing damage, as widely reported in the literature, the sensitivity of the nonlinear parameter is more than one order magnitude greater than linear ones. It is important to notice that without taking into account the "true" strain inside the sample, the net effect appears larger or does not really reflect the physics. A typical evaluation of strain amplitude is $v_{\text {laser }} / c$, $c$ being the speed of sound. This assumption is only valid in free space, it provides the strain component perpendicular to the surface, and does not hold for modal analysis. As an example, the speed of sound decreases with damage by $25 \%$ for most damaged samples which implies a $33 \%$ increase of strain for a given particle velocity level. Table 2 shows that the $C$ constant can increase by more than a factor of 10 which implies a 10 times increase of strain amplitude for a given particle velocity level. That means that with the previous approximation, being the slope of the trend in Eq.2, the nonlinearity is over estimated making the apparent sensitivity too high. Then, applying this assumption for modal analysis may lead to erroneous interpretations in case of non-symmetric mode shapes such as shown in FIG. 6.

\section{Conclusion and prospects}

In this paper the combination of linear measurements (RUS) with numerical simulation and nonlinear measurements (NRUS) is proposed in order to quantitatively evaluate the nonlinear behavior of materials. This paper highlights the importance of the mode shape to quantitatively evaluate the nonlinearity. It is applied to study the effect of thermal damage in concrete with results correlated to the evolution of the microstructure. The evolution of the Young's modulus and Poisson ratio evaluated from RUS are in agreement with the literature. 
The cement/aggregate interface (ITZ) is identified as the main source of nonlinearity for thermal damage regarding the thermo mechanical properties of concrete. The large sensitivity of the nonlinear parameter is shown with a sensitivity two orders of magnitude higher than a standard ultrasonic nondestructive indicator such as pulse wave velocity.

At present time, many studies emphasize the nonlinear $\alpha$ parameter to be highly sensitive to damage, which is true. However, due to the lack of the "true" strain in the sample, most of them are not comparable to each other, thus making impossible the proper validation of the data. The presented methodology is one of the possible ways to extend the literature with quantitative values comparable from one material to another by providing absolute values of $\alpha$, rather than simply relative changes.

The assessment of gradual damage in depth is one of the main problems in Nuclear Energy concrete structure subject to both radiation and elevated temperature. It is important to underline that this procedure applies for any kind of geometry (cores or parallelepiped) with any aspect ratio. So, by considering core extraction from concrete structure, one can cut these cores into slices corresponding to various depths and apply the present methodology to evaluate the depth and the amount of damage. In contrast to standard mechanical tests (e.g., stress-strain testing) that only apply to standard cores (i.e., specific dimensions), thus making it impossible to get the evolution in depth of linear and nonlinear properties in that case.

Studies in progress are aimed at going one step beyond these quantitative nonlinearity values in order to nondestructively provide the evolution of elastic properties with strain amplitude, i.e., being able to provide the evolution of the Young's modulus and Poisson ratio as a function of strain amplitude.

\section{AKNOWELDGEMENTS}

Authors thank the US DOE Used Fuel Disposition (Storage) campaign and the Electrical Power Research Institute for supporting this study and Payan's visit to LANL, and CSTB for manufacturing the samples. Payan was also supported by Aix Marseille Univ. Authors would also like to thank anonymous reviewers for significant suggestions as well as James Ten Cate, Paul Johnson, and Robert Guyer for useful discussion during the course of this work. 


\section{References}

${ }^{1} \mathrm{P}$. Nagy, Fatigue damage assessment by nonlinear ultrasonic materials characterization, Ultrasonics 36, 375-381 (1998)

${ }^{2}$ C. Payan, V. Garnier, J. Moysan and P. A. Johnson, Applying nonlinear resonant ultrasound spectroscopy to improving thermal damage assessment in concrete, Journal of the Acoustical Society of America 121 (4), EL125-EL130 (2007)

${ }^{3}$ A. Menou, Thermal and mechanical behavior of concrete, a Multi-Scale Approach to Thermal Damage: Applications to Concrete at High Temperature, PhD Thesis, University Pau et Pays de l'Adour, France, 2004 (in French)

${ }^{4}$ A.M. Neville, Properties of Concrete, 3rd ed. (Longman Scientific and Technical, Essex, England, 1981), 1-779.

${ }^{5}$ Y.F. Fu, Y.L. Wong, C.A. Tang, C.S. Poon,Thermal induced stress and associated cracking in cement-based composite at elevated temperatures-Part I: Thermal cracking around single inclusion, Cement \& Concrete Composites 26 (2), 99-111 (2004)

${ }^{6}$ Y.F. Fu, Y.L. Wong, C.A. Tang, C.S. Poon,Thermal induced stress and associated cracking in cement-based composite at elevated temperatures-Part II: thermal cracking around multiple inclusions, Cement \& Concrete Composites 26 (2), 113-126 (2004)

${ }^{7}$ D. Landau and E. M. Lifshitz, Theory of Elasticity, 3rd (revised) English ed. (Pergamon, New York, 1986), 1-187

${ }^{8}$ R. Guyer and P. A. Johnson, Nonlinear mesoscopic elasticity: Evidence for a new class of materials, Physics Today 52, 30-35 (1999)

${ }^{9}$ K. Van Den Abeele, P.A. Johnson, and A. Sutin, "Nonlinear Elastic Wave Spectroscopy (NEWS) techniques to discern material damage. Part I: Nonlinear Wave Modulation Spectroscopy (NWMS)," Res. Nondestruct. Eval. 12, 17-30 (2000).

${ }^{10} \mathrm{~K}$. Van Den Abeele and J. De Visscher, Damage assessment in reinforced concrete using spectral and temporal nonlinear vibration techniques. Cem. Concr. Res. 30, 1453-1464 (2000)

${ }^{11}$ M. Bentahar, H. El Aqra, R. El Guerjouma, M. Griffa, and M. Scalerandi, "Hysteretic elasticity in damaged concrete: Quantitative analysis of slow and fast dynamics," Phys. Rev. B 73, 014116 (2006)

${ }^{12}$ F. Bouchaala, C. Payan, V. Garnier and J.P. Balayssac, Carbonation Assessment in Concrete by Nonlinear Ultrasound, Cem. Concr. Res. 41, 557-559 (2011)

${ }^{13}$ K.J. Lesnicki, J.-Y. Kim, K.E. Kurtis, and L.J. Jacobs, Characterization of ASR Damage in Concrete using Nonlinear Impact Resonance Acoustic Spectroscopy Technique, NDT\&E Int. 44, 721-727 (2011)

${ }^{14}$ K. Van Den Abeele, J. Carmeliet, J.A. TenCate, and P.A. Johnson, "Nonlinear Elastic Wave Spectroscopy (NEWS) techniques to discern material damage. Part II: Single mode nonlinear resonance acoustic spectroscopy," Res. Nondestruct. Eval. 12, 31-43 (2000).

${ }^{15}$ P. Johnson and A. Sutin, Slow dynamics and anomalous nonlinear fast dynamics in diverse solids, J. Acoust. Soc. Am. 117, 124-130 (2005)

${ }^{16}$ A. Migliori, John L. Sarrao, Resonant ultrasound spectroscopy: applications to physics, materials measurements, and nondestructive evaluation, Wiley, 1997

${ }^{17}$ T.J. Ulrich, K.R. McCall and R. Guyer, Determination of elastic moduli of rock samples using resonant ultrasound spectroscopy, J. Acoust. Soc. Am. 111(4), 1667-74 (2002).

${ }^{18}$ W. Wu et al., Measurement of Mechanical Properties of Hydrated Cement Paste Using Resonant Ultrasound Spectroscopy, Journal of ASTM International, Vol. 7, No. 5 (2010)

${ }^{19}$ S. Bernard S, Q. Grimal, P. Laugier, Accurate measurement of cortical bone elasticity tensor with resonant ultrasound spectroscopy, J. Mech. Behav. Biomed. Mater. 18, 12-19 (2013).

${ }^{20}$ J. Xiao and G. König, "Study of Concrete at High Temperature in China-An Overview,"

Fire Safety Journal 39, 89-103 (2004). 
${ }^{21}$ U. Schneider, C. Diererichs, and C. Ehm, "Effect of Temperature on Steel and Concrete for PCRV's," Nuclear Engineering and Design 67, 245-258 (1981).

${ }^{22}$ J.C. Maréchal, Variations of the modulus of elasticity and Poisson's ratio with temperature, Concrete for Nuclear Reactors, ACI SP-34, 1, $495-503$ (1972)

${ }^{23}$ P.A. Johnson, B. Zinszner, P. Rasolofosaon, F. Cohen-Tenoudji, K. Van Den Abeele,

Dynamic measurements of the nonlinear elastic parameter in rock under varying conditions, J. Geophys. Res. 109, B02202 (2004).

${ }^{24}$ K. Van Den Abeele, J. Carmeliet, P.A. Johnson, and B. Zinzsner, The influence of water saturation on the nonlinear mesoscopic response of earth materials, and the implications to the mechanism of nonlinearity, J. Geophys. Res. 107 (B6), 2121 (2002).

${ }^{25}$ S. Haupert, G. Renaud, J. Rivière, M. Talmant, P.A. Johnson, and P. Laugier, "Highaccuracy acoustic detection of non-classical component of material nonlinearity," J. Acoust. Soc. Am. 130(5), 2654-2661 (2011).

${ }^{26}$ H. Ramanasse, C. Payan, V. Garnier, N. Chaouch, B. Lombard, N. Favrie, Influence of the microstucture on the Nonlinear properties of plaster : Experimental evidence and numerical simulation, $18^{\text {th }}$ International Conference on Nonlinear Elasticity in Materials, Ascona, Switzerland, June $9-14^{\text {th }}$ (2013)

${ }^{27}$ M. Scalerandi, M. Griffa, P. Antonaci, M. Wyrzykowski and P. Lura, Nonlinear elastic response of thermally damaged consolidated granular media, J. Appl. Phys. 113, 154902 (2013) 


\section{Figures Caption}

FIG. 1. NRUS curves for (a) optimal and (b) more complex geometry.

FIG. 2. Schematic of rectangular concrete block sample sitting on the conical transducer stand.

FIG. 3. (Color Online) Resonance spectrum taken from sample OC-120. ' $\mathrm{x}$ ' indicates peak values used in RUS inversion.

FIG. 4. (Color Online) NRUS experimental scheme.

FIG. 5. (Color Online) Resonance modes of the Mortar samples set. The sample is also shown, including the transducer and the laser spot location (spot at the sample center).

FIG. 6. (Color Online) Experimental and simulated resonance modes for M20 sample. The curve is the experimental spectrum, the vertical lines are the simulated resonant frequencies (limited to the most three energetic ones). The corresponding mode shapes are also shown.

FIG. 7. (Color Online) Data acquisition procedure. Left panel: generation. Right panel: reception

FIG. 8. Plexiglas sample NRUS results

FIG. 9. (Color Online) Evolution of elastic properties for the full set of sample as a function of thermal damage.

FIG. 10. (Color Online) High Performance Concrete NRUS curves

FIG. 11. (Color Online) Ordinary Concrete NRUS curves

FIG. 12. (Color Online) Mortar NRUS curves

FIG. 13. (Color Online) Result's compilation of nonlinear parameters.

FIG. 14. (Color Online) Sensitivity study. PWV is the Pulse Wave Velocity evaluated from Time Of Flight measurements. 


\section{List of tables}

Table 1. Concrete samples composition and codification

\begin{tabular}{|l|l|l|l|}
\hline Composition $\left(\mathrm{kg} / \mathrm{m}^{3}\right)$ & $\begin{array}{l}\text { High Performance } \\
\text { Mortar }(\mathrm{M}) \\
\text { w/c }=0.3\end{array}$ & $\begin{array}{l}\text { High Performance } \\
\text { Concrete }(\mathrm{HPC}) \\
\text { w/c }=0.3\end{array}$ & $\begin{array}{l}\text { Ordinary Concrete } \\
(\mathrm{OC}) \\
\text { w/c }=0.5\end{array}$ \\
\hline Codification & M 20-120-250-400 & HPC 20-120-250-400 & OC 20-120-250-400 \\
\hline Limestone 12.5-20 & 0 & 579 & 514 \\
\hline Limestone 5-12.5 & 0 & 465 & 514 \\
\hline Limestone 0-5 & 739 & 442 & 401 \\
\hline Seine 0-4 & 727 & 435 & 401 \\
\hline Silica fume & 37 & 22 & 0 \\
\hline CPA 52.5 cement & 602 & 0 & 0 \\
\hline CEM I 52.5 cement & 0 & 360 & 0 \\
\hline CEM I 32.5 cement & 0 & 0 & 350 \\
\hline Water & 219 & 136 & 181 \\
\hline GT Super plasticizer & 10 & 12 & 0 \\
\hline
\end{tabular}


Table 2. Comparison of numerical and experimental data and values of the $\mathrm{C}$ constant.

\begin{tabular}{|c|c|c|c|c|}
\hline$T^{\circ}\left({ }^{\circ} \mathrm{C}\right)$ & $\begin{array}{l}\text { Experiments } \\
f_{0}(\mathrm{kHz})\end{array}$ & Simulation $f_{0}(\mathrm{kHz})$ & Error $(\%)$ & C. $10-3(\mathrm{~s} / \mathrm{m})$ \\
\hline \multicolumn{5}{|c|}{ High Performance Concrete } \\
\hline 20 & 24.41 & 24.29 & 0.481 & 0.269 \\
\hline 120 & 23.78 & 23.56 & 0.921 & 0.34 \\
\hline 250 & 22.3 & 22.55 & 1.115 & 0.291 \\
\hline 400 & 17.45 & 16.9 & 3.152 & 2.1 \\
\hline \multicolumn{5}{|c|}{ Ordinary Concrete } \\
\hline 20 & 22.14 & 21.76 & 1.738 & 0.374 \\
\hline 120 & 21.72 & 21.31 & 1.881 & 0.469 \\
\hline 250 & 19.4 & 18.73 & 3.444 & 1.17 \\
\hline 400 & 15.43 & 14.08 & 8.745 & 11.5 \\
\hline \multicolumn{5}{|l|}{ Mortar } \\
\hline 20 & 22.15 & 22.1 & 0.224 & 0.506 \\
\hline 120 & 22.2 & 22.33 & 0.566 & 0.464 \\
\hline 250 & 21 & 20.87 & 0.587 & 0.745 \\
\hline 400 & 19.34 & 18.28 & 5.478 & 1.77 \\
\hline
\end{tabular}

